METZLER KOMPONISTEN LEXIKON 


\section{METZLER \\ KOMPONISTEN LEXIKON}

340 werkgeschichtliche Porträts

Mit 313 Abbildungen

Herausgegeben von Horst Weber

Verlag J.B. Metzler Stuttgart · Weimar 


\title{
Inhaltsverzeichnis
}

\author{
Vorwort Seite $V$ \\ Hinweise zur Benutzung Seite VI \\ Verzeichnis der Abkürzungen und Siglen Seite VII \\ Komponisten von A-Z Seiten 1-899 \\ Verzeichnis der Autorinnen und Autoren Seite 900 \\ Weiterführende Literatur Seite 903 \\ Personenregister Seite 909 \\ Bildnachweise Seite 919
}

\begin{abstract}
Die Deutsche Bibliothek - CIP-Einheitsaufnahme
Metzler-Komponisten-Lexikon: 340 werkgeschichtliche Porträts / hrsg. von Horst

Weber. - Stuttgart ; Weimar : Metzler, 1992

ISBN 978-3-476-0o847-3

NE: Weber, Horst [Hrsg.]; Komponisten-Lexikon
\end{abstract}

\author{
ISBN 978-3-476-00847-3 \\ ISBN 978-3-476-03421-2 (eBook) \\ DOI 10.1007/978-3-476-03421-2
}

Dieses Werk einschließlich aller seiner Teile ist urheberrechtlich geschützt. Jede Verwertung außerhalb der engen Grenzen des Urheberrechtsgesetzes ist ohne

Zustimmung des Verlages unzulässig und strafbar. Das gilt insbesondere für Vervielfältigungen, Übersetzungen, Mikroverfilmungen und die Einspeicherung und Verarbeitung in elektronischen Systemen.

(C) 1992 Springer-Verlag GmbH Deutschland

Ursprünglich erschienen bei J. B. Metzlersche

Verlagsbuchhandlung und Carl Ernst Poeschel

Verlag GmbH in Stuttgart 1992 


\section{Vorwort}

Dieses Lexikon informiert über das Komponieren der Komponisten. In werkgeschichtlichen Portraits werden Aspekte wie stilistische Entwicklung, Traditionen der Gattung und des Tonsatzes, ästhetische Konzeptionen und Wirkungsgeschichte der Werke skizziert - je nach Komponist in wechselnder Auswahl oder Akzentuierung. Die Darstellung eines Lebenswerkes erstrebt nicht Vollständigkeit, sondern Charakteristik. Die üblicherweise vorherrschenden Informationen zur Biographie fließen nur insoweit ein, als sie zum Verständnis des Werkes notwendig sind.

Jedem Nachschlagewerk liegen Werturteile zugrunde, auch wenn sie häufig nicht thematisiert werden, weil dies die lexikalische Objektivität zu trüben scheint. Aber diese sind es - wenn nicht eine unreflektierte lexikographische Tradition -, welche die Auswahl und Gewichtung der Artikel jedes Lexikons begründen. Zwei Gesichtspunkte waren für den Herausgeber vorrangig: eine internationale Breite der Auswahl, und zwar für alle Epochen, denn nie - nicht einmal zur Zeit der ' Wiener Klassik ‘ - war der deutsche Sprachraum der Nabel der musikalischen Welt; sodann ein entschiedener Schwerpunkt auf der Musik des 20. Jahrhunderts, die insofern besonders der Information bedarf, als in ihr verbindliche Normen zusehends verfallen und an deren Stelle eine je persönliche Aneigung von Tradition und die Gewinnung eines eigenen ästhetischen Standpunkts getreten sind. Hinzu kamen als entscheidende Kriterien künstlerische Qualität und historische Bedeutung, die ja keineswegs immer zusammenfallen. Es wurde auch die Gelegenheit genutzt, auf heute Entlegeneres, das zu Unrecht vergessen scheint, aufmerksam zu machen, und manchmal auch, auf (allzu) Bekanntes zu verzichten. Die Präsenz eines Komponisten im Musikbetrieb, gegen dessen Strich die Auswahl gebürstet ist, kam nur als zusätzlicher Gesichtspunkt in Betracht, denn ein Lexikon hat eine andere Funktion als ein Konzert- oder Opernführer.

Eine pragmatische Entscheidung war der Verzicht auf Komponisten der sogenannten populären Musik (mit Ausnahme einiger 'Stammväter`). Damit soll nicht der - problematischen - Trennung von E- und U-Musik das Wort geredet, sondern der Tatsache Rechnung getragen werden, daß in der populären Musik die Fluktuation von Trends und Musikern, die häufig gleichermaßen Komponisten wie Interpreten sind, stärker ist als in der sogenannten E-Musik, also der unvermeidliche Alterungsprozeß der Informationen schneller voranschreitet. Der Verlag hat daher die Publikation eines eigenen Lexikons der populären Musik ins Auge gefaßt.

Der Herausgeber dankt allen Autorinnen und Autoren für ihre Beiträge, dem Verlag für die sorgsame Betreuung des Buches, Dieter Thoma und Elke Rühl für ihre Hilfe bei der Korrektur, sowie seinen Kollegen an der Folkwang-Hochschule Essen - insbesondere Matthias Brzoska, Wolfgang Grandjean, Nicolaus A. Huber und Claus Raab - für mancherlei Entlastung, Rat oder Hilfe. 


\section{Hinweise zur Benutzung}

Im Titelkopf ist der erste Vorname der Rufname, sofern er nicht in Klammern steht. Innerhalb des Textes werden Komponisten, die mit einem Artikel in diesem Lexikon vertreten sind, nur mit ihrem Zunamen, alle anderen Personen dagegen bei ihrer ersten Erwähnung mit Vor- und Zunamen genannt. Der Leser ist dadurch ohne Querverweise über die Stichwortauswahl informiert und kann entscheiden, ob er sich durch die Lektüre anderer Artikel weitergehend informieren will. Querverweise stehen nur bei Sachbegriffen, die andernorts näher erläutert sind.

Namen und Titel, deren originaler Schreibweise die kyrillische Schrift zugrunde liegt, werden in Transliteration gemäß den Regeln der International Standard Organisation wiedergegeben, es sei denn der Komponist hat im Ausland eine Transkription seines Namens autorisiert (z. B. Stravinsky). Die Lebensdaten werden bis I 9 I 7 nach dem Julianischen (russischen) Kalender angegeben; die Datierung gemäß Gregorianischem Kalender steht in Klammern.

Einige Komponisten aus früher Zeit sind, allgemeinem Brauch in der Mediaevistik entsprechend, aber abweichend von anderen Musiklexika, unter ihrem Hauptnamen (der später der Vorname wurde) eingeordnet (z. B. Guillaume de Machaut).

Werknamen - nicht nur Titel, sondern auch Gattungsnamen - erscheinen im Text (nicht in den Literaturhinweisen) kursiv. Die Entstehung eines Werkes wird, sofern sie sich über mehrere Jahre erstreckte, durch Bindestrich (z. B. I 904-06), verschiedene Fassungen durch Schrägstrich (z. B. 1922/1947) angezeigt; bei Bühnenwerken sind stattdessen Ort und Jahr der Uraufführung genannt, die Entstehungszeit zusätzlich nur, wenn bis zur Uraufführung ein größerer Zeitraum verstrichen ist. Den Angaben zur Datierung sind gegebenenfalls die Übersetzung des Originaltitels, bei Vokalwerken meist auch der Name des Dichters bzw. Librettisten vorangestellt.

Zitate werden in der Regel durch Kurztitel nachgewiesen, die bibliographischen Angaben sind in den Literaturhinweisen am Ende des Artikels komplettiert. Ein Pfeil vor dem Kurztitel verweist auf einen Titel in der Weiterführenden Literatur am Ende des Lexikons.

Die Literaturhinweise sind knapp gehalten, sie beschränken sich auf ältere Standardwerke und neuere Literatur, berücksichtigen jedoch eine komplizierte Forschungslage (z. B. bei Pergolesi). Sie sind folgendermaßen gegliedert:

[D] Notenausgaben (Gesamtausgabe, sonst eine Auswahl möglichst verläßlicher Einzelausgaben) bzw. wichtige Verlage (vor allem bei zeitgenössischen Komponisten) Schriften, Memoiren - Briefe - Dokumente, Ausstellungskataloge

[op. Eigenständiges Werkverzeichnis, eventuell mit betreffender Literatur

$\Leftrightarrow$ Eigenständige Bibliographie

[7] Dem Komponisten gewidmete Periodica

Biographien und Studien (mit Hinweisen auf wichtige eingearbeitete Werkverzeichnisse und Bibliographien, vor allem aus neuerer Zeit)

Ergänzend zu den nachgewiesenen Werkverzeichnissen sei hier auf die Werkverzeichnisse in den großen Enzyklopädien (siehe Weiterführende Literatur), insbesondere in The New Grove Dictionary of Music and Musicians (London I 980) hingewiesen. 
Verzeichnis der Abkürzungen und Siglen

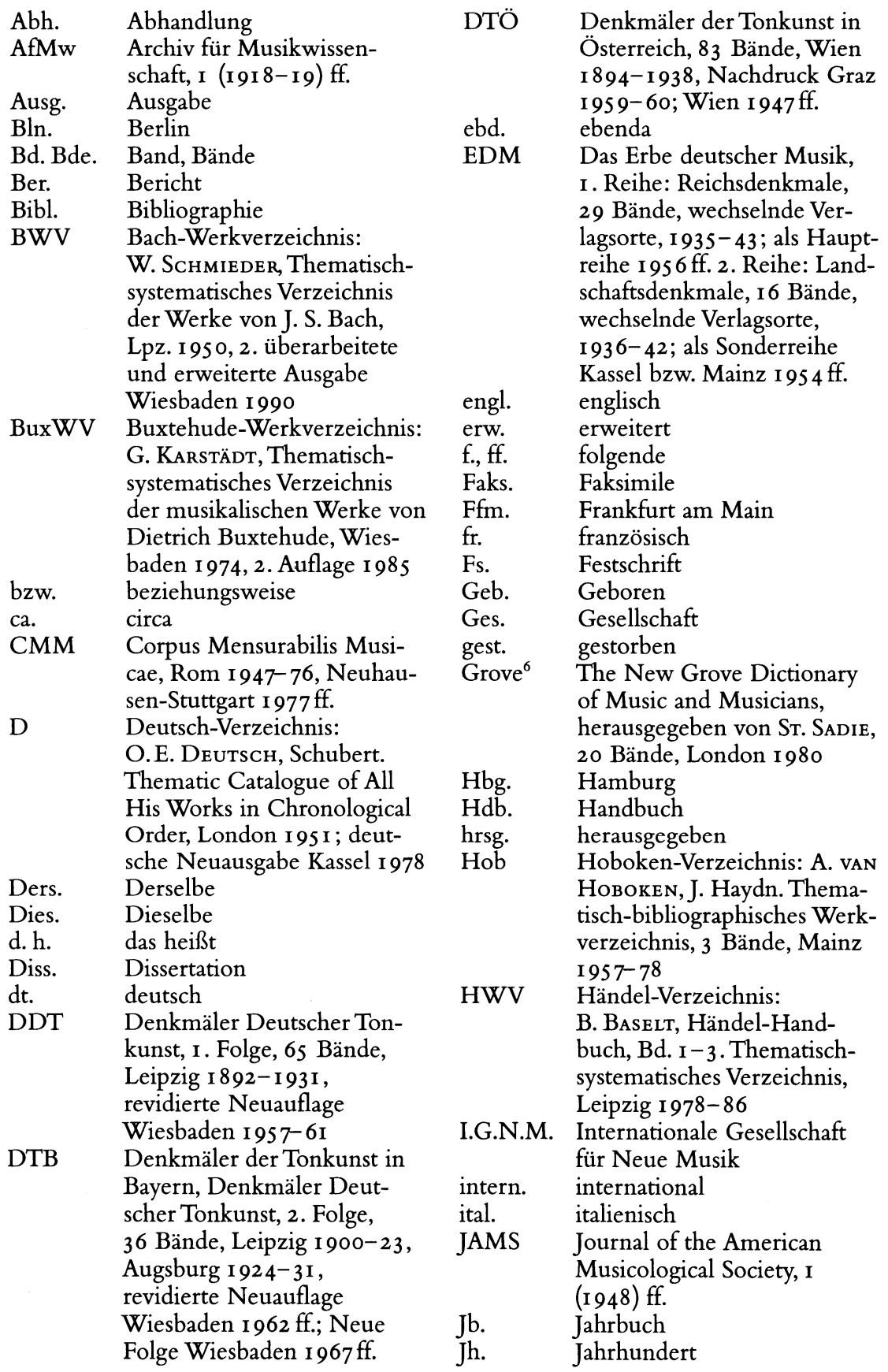




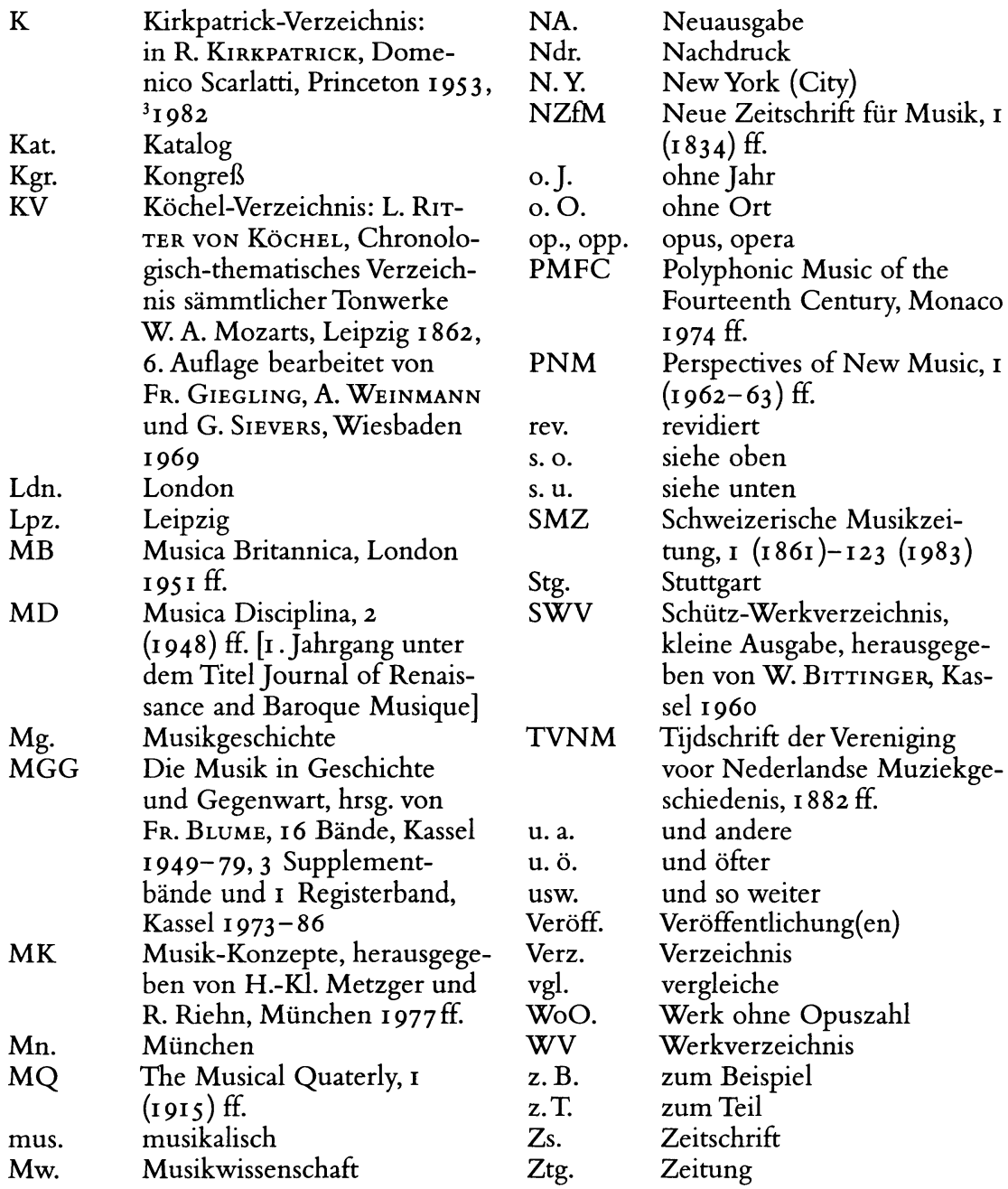

Zeitschriften sind ohne Titelvarianten und Unterbrechungen ihres Erscheinens genannt. 